\title{
CONSUMO DE FIBRAS DIETÉTICAS NO DIABETES MELLITUS: AVALIAÇÃO DO CONHECIMENTO E FREQUÊNCIA DE SUA INGESTÃO POR PACIENTES ATENDIDOS EM UMA UNIDADE DE SAÚDE ESCOLA DE SANTA CATARINA
}

\author{
CONSUMPTION OF DIETARY FIBERS IN DIABETES MELLITUS: \\ EVALUATION OF KNOWLEDGE AND FREQUENCY OF ITS INGESTION BY \\ PATIENTS ATTENDED IN A HEALTH UNIT SCHOOL OF SANTA CATARINA
}

Letícia De Oliveira Silva, Isabela Berger, Tatiana Mezadri, Rosana Henn, Claiza Baretta, Cristina Henschel De Matos*.

Universidade do Vale do Itajaí, Itajaí, SC, Brasil.

*matoscris@gmail.com

Recebido em: 28/11/2019; Aceito em: 20/11/2020.

\section{RESUMO}

O Diabetes Mellitus (DM) afeta 415 milhões de indivíduos no mundo e 14,3 milhões no Brasil. Sabe-se que alimentos ricos em fibras alimentares têm papel fundamental no controle da glicemia sanguínea. Avaliar a influência do conhecimento sobre a importância da ingestão de fibras e sua frequência de consumo no controle do DM. A população foi composta por diabéticos tipo 2 que frequentam uma Unidade de Saúde Escola de Itajaí/SC. A coleta de dados foi realizada por entrevista contendo questões referentes às características socioeconômicas, conhecimentos e frequência de consumo de fibras. $O$ controle do DM foi avaliado através da Hemoglobina Glicada (HbA1c). A população constituiu-se de 22 pessoas, sendo a maioria do sexo feminino $(n=15 ; 68 \%)$, com idade média de $60( \pm 9,3)$ anos, e com no máximo $9( \pm 3,4)$ anos de estudo. A média de consumo semanal de legumes e saladas foi de oito porções $( \pm 4,5)$, enquanto as frutas foram citadas em 14 vezes na semana e os cereais integrais, sete vezes. Quando questionados sobre a capacidade das fibras de prevenir e tratar o diabetes, $86 \% \quad(n=19)$ dos pacientes concordaram com a afirmação. Encontrou-se tempo médio de diagnóstico de 9 $( \pm 7)$ anos e média de HbA1c de $8 \%( \pm 2)$. Observou-se conhecimento insuficiente a respeito de fibras alimentares, mesmo sabendo da importância de consumi-las adequadamente, visto que a ingestão de legumes e saladas, de frutas e de cereais integrais foi avaliada como insuficiente, podendo tal fato estar relacionado com renda, ausência de cuidado com a saúde e tempo de diagnóstico.

Palavras-chave: Conhecimento. Diabetes mellitus. Dietoterapia. Fibras na dieta. 


\section{ABSTRACT}

Diabetes Mellitus (DM) affects 415 million individuals worldwide and 14.3 million in Brazil. It is known that foods which are rich in dietary fibers have a fundamental role in the control of blood glucose. The aim of the present study was to evaluate the influence of knowledge about the importance of fiber intake and its frequency of consumption in controlling DM. The population was composed of type 2 diabetic patients who attend a Health Unit School in Itajaí / SC. Data collection was done through an interview containing questions regarding socioeconomic characteristics, knowledge and frequency of fiber consumption. DM control was assessed using glycated hemoglobin (HbA1c). The population consisted of 22 people, most of them female $(n=15 ; 68 \%)$, with an average age of $60( \pm 9.3)$ years old, and a maximum of $9( \pm 3.4)$ years of background education. The weekly consumption average of vegetables and salads was eight servings $( \pm 4.5)$, while fruits were mentioned 14 times a week and whole grains, seven times. When asked about the capacity of fibers to prevent and treat diabetes, $86 \%(n=19)$ of the patients agreed with the statement. An average time of diagnosis of $9( \pm 7)$ years was found, as well as a $\mathrm{HbA1c}$ average of $8 \%( \pm 2)$. Insufficient knowledge about dietary fibers was observed, even knowing the importance of consuming them properly, since the consumption of vegetables and salads, fruits and whole grains was assessed as insufficient, which may be related to low income, lack of health care and time of diagnosis.

Keywords: Diabetes mellitus. Dietary fiber. Diet therapy. Knowledge.

\section{INTRODUÇÃO}

O diabetes mellitus (DM) é um conjunto de distúrbios metabólicos caracterizados por hiperglicemia, que ocorre por falhas na atuação e/ou secreção de insulina. A natureza crônica da doença, bem como a gravidade das complicações e os meios necessários para controlá-los, a tornam onerosa para os pacientes afetados e para o sistema de saúde, visto que afeta 415 milhões de indivíduos no mundo e 14,3 milhões no Brasil (LEMOS; PATEK; MEZZOMO, 2017).

São atribuídas ao DM complicações classificadas em agudas e crônicas, que podem ser acarretadas pelo tratamento inadequado ou pelos níveis glicêmicos descompensados. As complicações crônicas podem ainda ser subdivididas em micro e macrovasculares. Neuropatia, retinopatia, nefropatia e isquemia, são alguns dos agravamentos microvasculares mais comuns. Quanto aos macrovasculares, destacam-se 0 desenvolvimento de cardiopatia isquêmica, doença cerebrovascular e doença vascular periférica (ALMEIDA et al., 2017).

Sabe-se que a suscetibilidade genética exerce um papel importante na ocorrência do diabetes mellitus tipo 2 (DM2), porém a atual epidemia reflete o aumento da ingestão energética, redução da prática de atividades físicas, sobrepeso e obesidade (SBD 2017/2018). Deste modo, o conhecimento acerca 
das características da doença e sobre o tratamento nutricional nestes pacientes é fundamental para um melhor controle da patologia (OLIVEIRA et al., 2016).

O controle da doença pode ser feito por meio da associação de uma dieta alimentar adequada de baixo índice glicêmico, prática de exercício físico e uso de medicamentos hipoglicemiantes (ALMEIDA; SILVA; SOUSA, 2017). A alimentação de pacientes com DM2 preconiza a qualidade da alimentação em sua totalidade, já que o consumo de carboidratos deve incluir vegetais, frutas, grãos integrais e legumes, que são fontes de fibras (ZANETTI et al., 2015).

A ingestão de alimentos ricos em fibras alimentares apresenta efeitos benéficos no controle da glicemia e no metabolismo de lipídeos. Além disso, sua presença na alimentação é fundamental uma vez que diminuem o risco de várias doenças (SBD, 2017; 2018).

Portanto, a alimentação adequada é extremamente importante para estes pacientes, sendo utilizada como uma ferramenta no manejo do DM. Além disso, uma vez que a população diabética possui conhecimento acerca de como a dieta é capaz de interferir na doença, seu controle torna-se ainda mais efetivo. Diante disso, o objetivo do presente estudo foi avaliar a influência do conhecimento sobre fibras e sua frequência de consumo no controle do DM.

\section{MATERIAL E MÉTODOS}

Realizou-se um estudo observacional, descritivo e transversal, com abordagem quantitativa do tipo Survey.

A população foi constituída por pacientes portadores de DM2 atendidos no ambulatório de endocrinologia de uma Unidade de Saúde Escola de Itajaí/SC, entre abril e agosto de 2019 e os critérios de inclusão estabelecidos foram: adultos ( $\geq 20$ anos - 59 anos) e idosos ( $\geq 60$ anos), portadores de DM2, não insulino-dependentes, com exame de hemoglobina glicada registrado em prontuário e realizado nos últimos três meses.

O trabalho foi aprovado pelo Comitê de Ética em Pesquisa (CEP), sob protocolo 3.086.369, na data 16/12/2018.

A coleta de dados foi realizada pelas acadêmicas responsáveis pelo projeto com auxílio de questionário, aplicado em forma de entrevista àqueles que manifestaram interesse, após 0 aceite e assinatura do Termo de Consentimento Livre e Esclarecido (TCLE). Ressalta-se que os prontuários dos usuários abordados foram previamente consultados para verificar se os mesmos estavam dentro dos critérios de inclusão da pesquisa.

Foi aplicado um questionário semiestruturado, adaptado de Martinho (2011), contendo questões referentes à idade, escolaridade, sexo, hábitos de consumo alimentar, conhecimento sobre fibras alimentares (alimentos fontes de fibras, recomendações segundo a Organização Mundial da Saúde (OMS), relação entre a presença de casca nos alimentos e o teor de fibras, subdivisão em fibras solúveis e insolúveis), meios de divulgação e informação sobre o assunto, relação entre fibras e variedade de alimentos e, por fim, relação entre fibras e DM.

O controle de DM dos pacientes foi avaliado de acordo com o exame bioquímico de Hemoglobina Glicada (HbA1c), que constava em seus prontuários. Considerou-se adequado o controle de indivíduos com DM que 
apresentaram valores entre a $6,5 \%$ a $7 \%$, de acordo com a Sociedade Brasileira de Diabetes (SBD, 2017; 2018).

Os dados coletados na pesquisa foram analisados com auxílio dos programas Microsoft Excel®, e as respostas obtidas sobre o conhecimento a respeito de fibras, foram comparados com a literatura recente sobre o tema. Para análise estatística descritiva, as variáveis quantitativas contínuas foram expressas por meio de médias e desvio-padrão e as variáveis categóricas por meio de frequências absolutas e relativas. Para a análise inferencial aplicou-se a correlação de Spearman para dados não paramétricos, considerando a natureza das variáveis estudadas. As diferenças foram consideradas significantes em nível de $p<0,05$, para rejeição da hipótese de nulidade.

\section{RESULTADOS E DISCUSSÃO}

A amostra do estudo foi composta por 22 pessoas, com idade média de $60( \pm 9,3)$ anos e, em sua maioria, por indivíduos do sexo feminino $(n=15)$ totalizando $68 \%$.

A caracterização populacional do estudo é semelhante com a encontrada por Zanetti et al. (2015), ao avaliar a adesão às recomendações nutricionais e variáveis sociodemográficas de 1.406 pacientes adultos com $\mathrm{DM}$, na região sudeste do Brasil, obtendo média de idade de $62,4( \pm 11,8)$ anos e predomínio do sexo feminino (66,67\%). Segundo Botton, Cúnico e Strey (2017), o autocuidado e a saúde feminina, principalmente no que diz respeito à saúde sexual e reprodutiva das mulheres, naturalizou o acesso desse público à saúde. O sexo masculino, por sua vez, é historicamente educado para aversão ao cuidado, pois a busca pelos serviços de saúde poderia ser contrária à virilidade, força e independência. Entende-se, portanto, que este dado não está relacionado à maior prevalência de $\mathrm{DM} 2$ em mulheres, mas sim, à menor procura dos homens pelo serviço de saúde.

Em relação à escolaridade, a média obtida foi de $9( \pm 3,4)$ anos de estudo, o que equivale ao "ensino fundamental" completo. Este dado se assemelha com o que encontraram Carvalho e Chagas (2016) ao avaliar o consumo alimentar de 105 diabéticos atendidos na estratégia saúde da família em um município do Piauí. A maior parte da sua amostra, equivalente a 47,6\% $(\mathrm{n}=50)$, possuía o ensino fundamental completo. Nesse aspecto, Borba et al. (2019), ao avaliar o conhecimento sobre diabetes e atitude para o autocuidado de idosos na atenção primária em saúde no Nordeste do Brasil, atribuíram a baixa escolaridade como fator de risco para conhecimento insuficiente e atitude negativa quanto ao autocuidado.

Em relação aos hábitos de consumo, 64\% $(n=14)$ dos indivíduos alegou consumir pelo menos uma porção de legumes e saladas ao dia enquanto $18 \%$ $(n=4)$ relatou consumir duas porções. Os demais indivíduos referiram consumir zero, três, quatro e 21 porções por semana, respectivamente. Portanto, a média de consumo de legumes e saladas por semana, foi de oito porções $( \pm$ 4,5).

Da mesma maneira, no estudo de Zanchim e Kirsten (2018), onde avaliou-se os marcadores do consumo alimentar de 100 pacientes com DM2, o consumo de saladas cruas, legumes e verduras cozidas foi citado com 
frequência de sete vezes na semana pela maioria dos entrevistados, o que também caracteriza consumo menor que três porções ao dia, contrariando o recomendado. Tal recomendação, segundo o Guia Alimentar para a População Brasileira (BRASIL, 2008), deve-se consumir diariamente pelo menos três porções de legumes e verduras como parte das refeições. Em consentimento, a recomendação da SBD (2017/2018) é que o consumo varie de três a cinco porções de hortaliças cruas e cozidas por dia.

A frequência média de consumo de frutas foi de 14 vezes na semana $( \pm 7)$. Dos pacientes avaliados, $32 \%(n=7)$ relataram consumi-las 14 vezes, e 0 mesmo percentual referiu consumir 21 porções compostas por frutas na semana. Dezoito por cento $(n=4)$ alegou o consumo de sete porções em uma semana. Uma, duas, dez e 28 porções de frutas foram citadas com a mesma frequência, totalizando $18 \%(n=4)$.

O presente dado se assemelha ao estudo realizado por Lima et al. (2015), que constatou também que $23,68 \%$ dos pacientes entrevistados consomem menos do que 3 frutas por dia. Em consonância, a maioria dos entrevistados por Zanchim e Kirsten (2018) referiram consumo de frutas em sete vezes na semana, configurando frequência de, no máximo, uma porção diária.

Entretanto, de acordo com a SBD (2017/2018, pacientes diabéticos devem seguir um plano alimentar variado, com o consumo mínimo de duas a quatro porções de frutas ao dia.

A respeito do hábito de comer fora de casa, $86 \% \quad(n=19)$ dos entrevistados relatou não o possuir, o que está de acordo com o estudo de Bezerra et al. (2017), que com o objetivo de verificar a aquisição de alimentos para consumo fora do lar, utilizaram dados da Pesquisa de Orçamento Familiar 2008-2009 em uma amostra de 152.895 indivíduos. A obtenção de alimentos fora do lar se deu principalmente em lanchonetes e restaurantes e a frequência de compra foi maior em adultos e adolescentes, o que caracteriza a população idosa como a que menos faz uso desse serviço.

Em relação ao consumo de fast-food, a maioria relatou não o fazer $(n=18 ; 82 \%)$, seguido de $18 \%(n=4)$ que referiu o consumo em um dia da semana. Esse resultado se assemelhou ao encontrado por Gomes, Soares e Gonçalves (2016), que entrevistaram 990 idosos ( $\geq 60$ anos) não institucionalizados, habitantes da zona urbana de Pelotas/RS, com o intuito de identificar os fatores associados à baixa qualidade de suas dietas. Constatouse baixa frequência de consumo de fast foods, mesmo nos idosos classificados com dieta de baixa e intermediária qualidade. Segundo os autores, esse dado pode estar relacionado com a formação dos hábitos alimentares dos idosos, ocorrida em época cuja oferta e consumo desses alimentos eram menores. Ou, ainda, ao entendimento popular de que esses alimentos são menos digestivos e inapropriados para o consumo após determinada idade.

Esses achados são satisfatórios de acordo com o que preconiza o Guia Alimentar para a População Brasileira (BRASIL, 2014), que recomenda que se evite redes de fast food no ato de se alimentar.

Acerca dos cereais integrais, $59 \%(n=13)$ dos indivíduos relatou consumir sete porções na semana, seguido, curiosamente, por $23 \%(n=5)$ que referiram não consumir cereais integrais nenhuma vez na semana. Vinte e uma 
porções semanais de cereais integrais foram citadas por $9 \%(n=2)$ dos entrevistados, sucedido de três, referido por uma pessoa (4,5\%). Assim sendo, a média de consumo de cereais integrais foi de 7 porções semanais $( \pm 6)$.

Semelhante aos achados do presente estudo, baixas frequências de consumo também foram observadas em um estudo de Dos Santos (2017), no qual avaliou-se o hábito alimentar e estado nutricional de 30 idosos com DM2 atendidos na Atenção Básica em Saúde do Ceará, e observou-se média de consumo de cereais integrais de apenas quatro porções por semana. Entretanto, estes resultados não condizem com a recomendação da SBD (2017/2018), que enfatiza o consumo de três ou mais porções de cereais integrais por dia.

Os resultados obtidos após os questionamentos em relação ao conhecimento sobre as fibras alimentares, estão dispostos na Tabela 1.

Tabela 1 - Conhecimento sobre fibras alimentares por pacientes diabéticos atendidos em uma Unidade de Saúde Escola de Santa Catarina, entre abril e agosto de 2019, na cidade de Itajaí-SC

\begin{tabular}{|c|c|c|c|c|c|c|}
\hline \multirow[t]{2}{*}{ Questionamento } & \multicolumn{2}{|c|}{ Concordo } & \multicolumn{2}{|c|}{ Discordo } & \multicolumn{2}{|c|}{$\begin{array}{l}\text { Não soube } \\
\text { responder }\end{array}$} \\
\hline & $\mathbf{n}$ & $\%$ & $\mathbf{n}$ & $\%$ & $\mathbf{n}$ & $\%$ \\
\hline $\begin{array}{l}\text { 1."Apenas os alimentos de origem vegetal, } \\
\text { no estado natural, possuem fibras" }\end{array}$ & 8 & 36 & 9 & 41 & 5 & 23 \\
\hline $\begin{array}{l}\text { 2. "Os alimentos de origem animal como } \\
\text { carnes, ovos e laticínios não contêm fibras } \\
\text { (exceto se adicionadas)" }\end{array}$ & 7 & 32 & 8 & 36 & 7 & 32 \\
\hline $\begin{array}{l}\text { 3."De acordo com a organização mundial } \\
\text { de saúde, um adulto médio deve ingerir } 25 \mathrm{~g} \\
\text { de fibra por dia" }\end{array}$ & 3 & 13,5 & 3 & 13,5 & 16 & 73 \\
\hline $\begin{array}{l}\text { 4."Os alimentos integrais têm menos } \\
\text { quantidade de fibra do que os não integrais" }\end{array}$ & 2 & 9 & 17 & 77 & 3 & 14 \\
\hline $\begin{array}{l}\text { 5."As frutas com casca têm menos } \\
\text { quantidade de fibra do que as } \\
\text { descascadas" }\end{array}$ & 0 & 0 & 19 & 86 & 3 & 14 \\
\hline $\begin{array}{l}\text { 6."As fibras alimentares classificam-se em } \\
\text { solúveis e insolúveis" }\end{array}$ & 0 & 0 & 6 & 27 & 16 & 73 \\
\hline
\end{tabular}

Fonte: os autores.

$\mathrm{Na}$ questão 1, 41\% dos entrevistados discordam da afirmação, consentindo com o que foi achado por Martinho (2011) ao avaliar o conhecimento da população portuguesa sobre fibras alimentares, evidenciando que a maioria das pessoas acredita que não somente em seu estado natural os alimentos de origem vegetal possuem fibras.

As respostas para as questões 4 e 5 revelam que a maioria dos indivíduos consegue identificar onde as fibras estão mais presentes dentre os 
alimentos de origem vegetal, o que é positivo, já que segundo Signor et al. (2016), é mediante o processo de conhecimento que o usuário possui atitudes e comportamentos de promoção à saúde com ampliação das possibilidades de manejo da doença, reabilitação e tomada de decisões que favoreçam a sua qualidade de vida.

Entretanto, a questão 6 destaca o não conhecimento sobre a solubilidade das mesmas, o que pode ser um fator de impedimento ao tratamento dietoterápico correto, uma vez que os efeitos benéficos responsáveis pelo controle da glicemia sanguínea são decorrentes, principalmente, da ingestão de fibras solúveis, que diminuem a velocidade com que os carboidratos são digeridos (PONTES et al., 2018).

Quanto ao local onde os entrevistados encontram habitualmente informações sobre fibras alimentares, a maioria relatou os centros de saúde, sendo este também o local mais citado pelos participantes quando questionados sobre o meio de divulgação que consideram mais adequado para que se incentive o consumo de fibras alimentares.

Em concordância, Flores et al. (2016), ao avaliarem que tipo de orientação a população idosa de Pelotas/RS estava recebendo dos profissionais de saúde a respeito de hábitos saudáveis, concluiu que idosos que se consultaram em Unidades Básicas de Saúde receberam mais orientações a respeito desse tema, sendo isso confirmado também por Molmelstet et al. (2019), que avaliaram o conhecimento nutricional de uma equipe multiprofissional que atende pessoas com DM na Atenção Básica da cidade de Blumenau/SC, a compreensão da importância de escolhas alimentares fontes de fibras obteve destaque nas falas dos profissionais.

Os resultados obtidos sobre o conhecimento e a relação entre fibras e variedade de alimentos estão dispostos na Tabela 2.

Tabela 2 - Conhecimento sobre a relação entre fibras e variedade de alimentos por pacientes diabéticos atendidos em uma Unidade de Saúde Escola de Santa Catarina, entre abril e agosto de 2019, na cidade de Itajaí-SC

\begin{tabular}{|c|c|c|c|c|c|c|}
\hline \multirow[t]{2}{*}{ Questionamento } & \multicolumn{2}{|c|}{ Concordo } & \multicolumn{2}{|c|}{ Discordo } & \multicolumn{2}{|c|}{$\begin{array}{l}\text { Não soube } \\
\text { responder }\end{array}$} \\
\hline & $\mathbf{n}$ & $\%$ & $\mathbf{n}$ & $\%$ & $\mathbf{n}$ & $\%$ \\
\hline $\begin{array}{l}\text { 1."As fibras alimentares têm origem apenas nos } \\
\text { alimentos vegetais" }\end{array}$ & 5 & 23 & 12 & 54 & 5 & 23 \\
\hline $\begin{array}{l}\text { 2."As fibras alimentares têm origem apenas nos } \\
\text { alimentos animais" }\end{array}$ & 3 & 14 & 15 & 68 & 4 & 18 \\
\hline $\begin{array}{l}\text { 3."As fibras alimentares podem ter origem nos } \\
\text { alimentos vegetais e animais" }\end{array}$ & 11 & 50 & 3 & 14 & 8 & 36 \\
\hline $\begin{array}{l}\text { 4."Leguminosas, cereais e frutas são alimentos } \\
\text { muito ricos em fibras alimentares" }\end{array}$ & 16 & 73 & 3 & 14 & 3 & 14 \\
\hline $\begin{array}{l}\text { 5."O consumo de fibras alimentares é maior nas } \\
\text { zonas urbanas do que nas zonas rurais" }\end{array}$ & 5 & 23 & 14 & 64 & 3 & 14 \\
\hline
\end{tabular}

Fonte: os autores. 
As respostas para as questões 1,2 e 3 reforçam que a maioria dos pacientes acredita que as fibras alimentares podem ser encontradas tanto em alimentos de origem vegetal, quanto de origem animal, mesmo respondendo corretamente à questão 4. Resultado semelhante foi encontrado por Martinho (2011), onde $43 \%$ dos entrevistados acreditam que a origem das fibras seja animal e vegetal, o que demonstra falta de conhecimento da população a respeito do assunto em ambos os estudos.

Em relação à questão 5 , a maioria dos entrevistados acham que 0 consumo das fibras é maior nas zonas rurais que nas zonas urbanas. Este é um dado positivo, uma vez que transparece a possível relação estabelecida pelos pacientes entre fibras e alimentos de origem vegetal, já que estes são provindos da zona rural, o que está em concordância com o Ministério da Saúde (BRASIL, 2013) que afirma que os moradores da zona rural possuem uma alimentação com qualidade superior aos residentes na área urbana.

A partir da análise das tabelas, observa-se conhecimento insuficiente a respeito de fibras alimentares, principalmente no que diz respeito à origem alimentar das fibras, recomendação de ingestão diária e solubilidade das mesmas, o que foi encontrado num estudo realizado por Pace et al. (2006), em que avaliou-se o conhecimento de 84 indivíduos diabéticos a respeito da doença e a sua importância no processo de autocuidado, e obtiveram resultados que mostraram fragilidade no conhecimento sobre diabetes, causas e complicações para o manejo da doença entre os participantes, o que vai de encontro também com Rodrigues et al. (2012) que obtiveram resultado insatisfatório para compreensão e adoção de práticas de autocuidado, bem como baixa prontidão para o manejo da doença ao avaliar a relação entre conhecimento, atitude, escolaridade e tempo de doença em 123 indivíduos com DM, atendidos em uma Unidade Básica de Saúde de São Paulo. E além destes, Dias et al. (2018), ao avaliarem o nível de conhecimento de pacientes diabéticos sobre DM2, obteve classificação "ruim" para a maioria dos seus entrevistados.

Quando questionados sobre a capacidade das fibras de prevenir e tratar o diabetes, $86 \% \quad(n=19)$ dos pacientes concordaram com a afirmação. Em relação a isso, sabe-se que as fibras solúveis auxiliam no controle da glicemia sanguínea, sendo benéficas para auxiliar no tratamento e prevenção do DM2 (SBD, 2017/2018). Portanto, as respostas demonstram que, de forma geral, os entrevistados reconhecem a importância do consumo adequado das fibras, entretanto não consomem em quantidade suficiente (segundo resultados obtidos na frequência alimentar).

$\mathrm{Na}$ tentativa de justificar o consumo insuficiente de fibras, Silva et al. (2019), ao avaliar a elevada prevalência de inadequação do consumo de fibras alimentares e os fatores associados em 1509 idosos do município de Campinas, concluiu que $90,1 \%$ da população estudada apresentava ingestão inadequada e possuía renda inferior a um salário mínimo. Em concordância, Borges et al. (2015), ao avaliar qual o custo de uma dieta saudável para famílias brasileiras de baixa renda, baseados na Pesquisa de Orçamentos Familiares realizada em 55.970 domicílios, encontraram que as aquisições de frutas, hortaliças e cereais não alcançaram as recomendações. Para isso, 
aumentaria os gastos com a alimentação em 39\% para indivíduos com renda menor que um salário mínimo, comprometendo em 145\% a renda familiar.

Outro possível motivo capaz de explicar o baixo consumo de fibras, seria a ausência do cuidado com a própria saúde por parte dos idosos, como foi encontrado por Belon et al. (2016), ao avaliar o perfil sócio-demográfico e uso de serviços de saúde em 1.949 idosos diabéticos de São Paulo, que encontraram $25 \%$ da sua população referindo-se não visitar o médico ou serviço de saúde periodicamente por causa da doença, sendo o principal motivo "não achar necessário".

Referente ao controle da doença, encontrou-se tempo médio de diagnóstico de $9( \pm 7)$ anos e a média de HbA1c encontrada foi de $8 \%( \pm 2)$, observando-se uma correlação estatisticamente significativa entre as duas variáveis $(r=0,4724 ; p=0,00264)$, ou seja, quanto maior o tempo de diagnóstico, maior o valor de hemoglobina glicada encontrado. Rodrigues et al. (2012), que obtiveram tempo médio de diagnóstico de 11 anos, relatam que quanto maior o tempo de diagnóstico menor será a prevalência de adesão ao tratamento dos usuários e maior o risco de complicações advindas de um insatisfatório controle metabólico. No mesmo sentido, Assunção (2017), ao avaliar o conhecimento e a atitude de 353 pacientes com DM da Atenção Primária em Saúde de Minas Gerais, os participantes apresentaram valores médios de HbA1C de 8,67\%. No mesmo estudo, o conhecimento da maioria dos usuários sobre diabetes foi avaliado como insatisfatório e, em relação à atitude de enfrentamento, observou-se que $98 \%$ possuíam atitude negativa acerca da doença.

Santos (2018), realizou estratégias educativas com 238 pacientes portadores de DM2, atendidos na Atenção Primária à Saúde de Divinópolis/MG. Na educação em grupo, observou-se melhoras significativas em relação à hemoglobina glicada, empoderamento e adesão ao autocuidado, confirmando compatibilidade entre conhecimento acerca da doença, consciência individual e menores níveis de hemoglobina glicada, o que reforça a importância da realização de atividades de educação em saúde como possível solução para a ausência do autocuidado.

De encontro ao exposto, Lima, Menezes e Peixoto (2018) encontraram diminuição da HbA1c, glicemia capilar, IMC e circunferência da cintura, ao realizar intervenção educativa baseada na teoria social cognitiva com 83 diabéticos cadastrados em uma Unidade de Estratégia de Saúde da Família de Goiás.

\section{CONCLUSÃO}

Diante dos resultados encontrados no presente estudo observou-se conhecimento insuficiente a respeito de fibras alimentares, principalmente no que diz respeito à origem alimentar das fibras, recomendação de ingestão diária e solubilidade das mesmas.

Apesar de os entrevistados saberem da importância do consumo adequado de fibras, constatou-se ingestão insuficiente de legumes e saladas, de frutas e de cereais integrais, o que pode estar relacionado com a renda, ausência de cuidado com a saúde em virtude do tempo de diagnóstico. 
De acordo com os entrevistados, os centros de saúde são os principais lugares onde se encontram informações sobre fibras, reforçando-se a importância da realização de atividades de educação alimentar e nutricional nestes locais, já que são o principal meio de acesso à informação para o público estudado.

Ressalta-se que o número reduzido de participantes foi um fator limitante do estudo, o que pode ser explicado pelo grande número de pacientes com DM2 já insulinodependentes, bem como portadores de DM1 ou de outras doenças endócrinas.

Por fim, sugere-se mais estudos que avaliem o conhecimento de fibras por pacientes diabéticos e seu consumo, cujos resultados possam direcionar à otimização da qualidade dos serviços de saúde, bem como identificar as demandas de educação alimentar e nutricional necessárias para atender essa população específica, uma vez que, conscientizar os pacientes e empoderá-los a respeito do tratamento, impactará diretamente nos gastos com saúde pública e superlotação dos serviços de saúde.

\section{REFERÊNCIAS}

ALMEIDA, L. B. C.; SILVA, R. C. C.; SOUSA, F. C. A. Functional foods in the management of diabetes mellitus type 2: bibliographic application. Facema, $v$. 3, n. 4, p. 727-731, 2017.

ALMEIDA, V. C. D. et al. Micro and macro vascular complications in people with type 2 diabetes mellitus in outpatient care. Revista Rene, v. 18, n. 6, p. 787793, 2017.

ASSUNÇÃO, S. C. et al. Knowledge and attitude of patients with diabetes mellitus in Primary Health Care. Escola Anna Nery, v. 21, n. 4, p. 1-7, 2017.

BELON, A. P. et al. Diabetes em idosos: perfil sócio-demográfico e uso de serviços de saúde. In: XVI Encontro Nacional de Estudos Populacionais. Minas Gerais: ABEP, 2016, p. 1-10.

BEZERRA, I. N. et al. Consumo de alimentos fora do lar no Brasil segundo locais de aquisição. Revista de Saúde Pública, v. 51, n. 15, p. 1-8, 2017.

BORBA, A. K. O. T. et al. Conhecimento sobre o diabetes e atitude para o autocuidado de idosos na atenção primária à saúde. Ciência \& Saúde Coletiva, v. 24, n. 1, p. 125-136, 2019.

BORGES, C. A. et al. Quanto custa para as famílias de baixa renda obterem uma dieta saudável no Brasil? Cadernos Saúde Pública, v. 31, n. 1, p. 137148, 2015.

BOTTON, A.; CÚNICO, S. D.; STREY, M. N. Diferenças de gênero no acesso aos serviços de saúde: problematizações necessárias. Mudanças-Psicologia da Saúde, v. 25, n. 1, p. 67-72, 2017. 
BRASIL. Ministério da Saúde. Secretaria de Atenção à Saúde. Departamento de Atenção Básica. Política Nacional de Alimentação e Nutrição / Ministério da Saúde, Secretaria de Atenção à Saúde. Departamento de Atenção Básica. 1. ed., 1. reimpr. - Brasília: Ministério da Saúde, 2013. 84 p.

BRASIL. Ministério da Saúde. Secretaria de Atenção à Saúde. Guia alimentar para a população brasileira: promovendo a alimentação saudável / Ministério da Saúde, Secretaria de Atenção à Saúde. Brasília: Ministério da Saúde, 2008. $210 \mathrm{p}$.

BRASIL. Ministério da Saúde. Secretaria de Atenção à Saúde. Departamento de Atenção Básica. Guia alimentar para a população brasileira / Ministério da Saúde, Secretaria de Atenção à Saúde, Departamento de Atenção Básica. 2. ed., 1. reimpr. - Brasília: Ministério da Saúde, 2014. 156 p.

CARVALHO, R. R. S.; DAS CHAGAS, L. R. Consumo alimentar em diabéticos atendidos na Estratégia Saúde da Família em município do Piauí. Revista Interdisciplinar, v. 9, n. 2, p. 97-106, 2016.

DIAS, S. M. et al. Níveis de conhecimento de pacientes diabéticos sobre a Diabetes Mellitus tipo II. Revista Interdisciplinar, v. 11, n. 3, p. 14-21, 2018.

FLORES, T. R. et al. Hábitos saudáveis: que tipo de orientação a população idosa está recebendo dos profissionais de saúde? Revista Brasileira de Epidemiologia, v. 19, n. 1, p. 167-180, 2016.

GOMES, A. P.; SOARES, A. L. G.; GONÇALVES, H. Baixa qualidade da dieta de idosos: estudo de base populacional no sul do Brasil. Ciência \& Saúde Coletiva, v. 21, n. 11, p. 3417-3428, 2016.

LEMOS, K. H.; PATEK, T. C.; MEZZOMO, T. R. Determinação do índice glicêmico e da carga glicêmica de dietas hospitalares servidas para diabéticos. Scientia Medica, v. 27, n. 4, p. 2-8, 2017.

LIMA, L. A. et al. Food habits of hypertensive and diabetics cared for in a Primary Health Care service in the South of Brazil. Revista de Nutrição, v. 28, n. 2, p.197-206, 2015.

LIMA, C. R.; MENEZES, I. H. C. F.; PEIXOTO, M. R. G. Educação em saúde: avaliação de intervenção educativa com pacientes diabéticos, baseada na teoria social cognitiva. Ciência \& Educação (Bauru), v. 24, n. 1, p. 141-156, 2018.

MARTINHO, C. A. C. Estudo sobre o conhecimento da população portuguesa acerca de fibras alimentares. 2011. 91f. Dissertação (Mestrado em Qualidade e Tecnologia Alimentar) - Instituto Politécnico de Viseu, Viseu, 2011. 
MOLMELSTET, K. C. et al. Conhecimento nutricional de uma equipe multiprofissional que atende pessoas com diabetes mellitus na atenção básica. Revista de Atenção Primária à Saúde, v. 19, n. 1, p. 31-38, 2019.

OLIVEIRA, L. M. S. M. et al. Adesão ao tratamento dietético e evolução nutricional e clínica de pacientes com diabetes mellitus tipo 2. HU Revista, v. 42, n. 4, p. 277-282, 2016.

PACE, A. E. et al. O conhecimento sobre diabetes mellitus no processo de autocuidado. Revista Latino-Americana de Enfermagem, v. 14, n. 5, p. 728734, 2006.

PONTES, E. et al. Os Benefícios do Consumo de Fibras Alimentares. International Journal of Nutrology, v. 11, n. 1, p. 324-327, 2018.

RODRIGUES, F. F. L. et al. Relação entre conhecimento, atitude, escolaridade e tempo de doença em indivíduos com diabetes mellitus. Acta Paulista de Enfermagem, v. 25, n. 2, p. 284-290, 2012.

SANTOS, L. M. et al. Avaliação do hábito alimentar e estado nutricional de idosos com diabetes mellitus tipo 2 atendidos na atenção básica de saúde do município de Porteiras-CE. Revista E-Ciência, v. 5, n. 1, p. 69-77, 2017.

SANTOS, J. C. Comparação das estratégias educativas em diabetes mellitus: educação em grupo e visita domiciliar. 2018. 135f. Dissertação (Mestradado em Enfermagem) - Universidade Federal de Minas Gerais, Belo Horizonte, 2018.

SIGNOR, F. et al. Conhecimento e educação em saúde de idosos portadores de diabetes mellitus. Fisioterapia Brasil, v. 17, n. 2, p. 171-175, 2016.

SILVA, G. M. et al. Elevada prevalência de inadequação do consumo de fibras alimentares em idosos e fatores associados: um estudo de base populacional. Revista Brasileira de Epidemiologia, v. 22, n. 1, p. 1-13, 2019.

SOCIEDADE BRASILEIRA DE DIABETES. Diretrizes da Sociedade Brasileira de Diabetes 2017-2018. Organização José Egídio Paulo de Oliveira, Renan Magalhães Montenegro Junior, Sérgio Vencio. -- São Paulo: Editora Clannad, 2017.

ZANCHIM, M. C.; KIRSTEN, V. R.; MARCHI, A. C. B. Marcadores do consumo alimentar de pacientes diabéticos avaliados por meio de um aplicativo móvel. Ciência \& Saúde Coletiva, v. 23, n. 12, p. 4199-4208, 2018.

ZANETTI, M. L. et al. Adherence to nutritional recommendations and sociodemographic variables in patients with diabetes mellitus. Revista da Escola de Enfermagem da USP, v. 49, n. 4, p. 619-625, 2015. 